\title{
Greek earthquake stirs controversy over claims for prediction method
}

London. An earthquake of 6.1 magnitude in southwestern Greece last week, which demolished two buildings and killed 20 people, has added fuel to a fierce scientific debate about a controversial method that claims to predict quakes by measuring

Panayotis Varotsos of the University of Athens, who developed the technique known as VAN - accuses Greek seismologists of "playing with people's lives" by denying the scientific validity of his method.

Using this method, he claims to have successfully predicted both the approximate timing and the location of the earthquake last week. He has now called on Greek seismologists to "drop their misguided opposition" to it.

Varotsos, who heads the solid state physics centre at the University of Athens, says that the Greek authorities were initially receptive to VAN when he correctly forecast a 6-magnitude earthquake close to Thessaloniki on 4 May and a 6.6-magnitude earthquake in northern Greece on 13 May.

The government, he claims, made a public declaration about the VAN method and promised to take countermeasures based on his predictions. But he says that the authorities reneged on their commitment when seismologists declared that the technique was scientifically unsound.

VAN is based on a discovery that Varotsos claims to have made in the late 1970 s that materials under stress emit characteristic electrical signals. After publishing this research in what he describes as "many leading physics journals", he decided to apply it increased electrical activity in the ground.

to earthquakes.

Monitoring stations exist at seven locations in Greece. Each consists of a network of electrode pairs designed to extract a clean electric field signal stripped of external 'noise' and ionospheric effects. Any unusual signal — such as a sudden burst of closely spaced peaks is taken as a sign that stresses are building up, and that an earthquake will take place within a specified period, usually three to six weeks.

Varotsos says that, using the VAN method, he has correctly predicted the timing, magni- Earthquake damage: how accurate are VAN predictions? tude and location of the three

most recent earthquakes in Greece. He has been faxing information on the predicted timing and magnitude of each earthquake to 29 seismological institutes worldwide. "How many more people will have to die before this method is recognized as being correct."

But many seismologists remain deeply sceptical, and say the method lacks the stamp of both scientific and statistical rigour. Xavier LePichon, of the Laboratoire de Géologie de l'Ecole Normale Supérieure in Paris, says Varotsos cannot claim success for VAN on a relatively limited number of observations.

David Booth of the British Geological Survey (but speaking in a personal capacity) says that VAN predictions on earthquake timings and locations, known as the 'prediction window', are "vague and loose", and

\section{Japan jumps on board the VAN wagon}

Tokyo. Japan is about to invest $¥ 250$ million (US $\$ 3$ million) in the controversial VAN technique for predicting earthquakes (see above). The Japan Meteorological Agency (JMA) has included $¥ 200$ million for VAN in a supplementary budget approved last month by the Diet to mitigate the effects of the Kobe earthquake.

The funds will be used to set up 20 detecting stations in the Kobe area to measure electric currents in the Earth near active faults. An additional seven will be established with wireless transmission of data for observing magnetic fields.

- Seiya Uyeda, a professor at both Tokai University and Texas A \& M University in the United States, and a leading proponent of VAN, says he welcomes the JMA funding. He helped to arrange a visit by
JMA scientists to Greece in mid-March to investigate the technique.

In addition to JMA funding, the Ministry of Education, Science and Culture has allocated about $¥ 20$ million in the supplementary budget to set up a VAN network at Hokkaido University. Uyeda's group will help to establish the stations in Hokkaido and the private Tokai University, will provide Uyeda's group with about $¥ 30$ million to "revitalize" its VAN research.

Japanese earthquake researchers have in general welcomed the decision to put money into VAN, even though there is still considerable scepticism about the technique. Man-made electrical noise from train lines and other sources make it difficult to observe electrical activity of natural

David Swinbanks origin in Japan. that earthquakes predicted by VAN have occurred later than expected and at different magnitudes. Even more significant, he says, is the fact that the connection between stresses in tectonic plates and electrical activity remains unproved.

A paper by four Greek seismologists that is being widely circulated says that Varotsos' prediction of the Thessaloniki earthquake was five days too late and two magnitude units too large.

But Varotsos is standing firm, and says that his 13 May prediction was of an earthquake that lies in an area "where no earthquake has taken place for 1,000 years".

Despite their doubts, not all seismologists dismiss VAN completely, and some are even beginning to investigate it further. Varotsos recently returned from a 10 -day trip to Japan where the government (see left) has promised to invest heavily in VAN.

Last month, 40 scientists attended a closed two-day meeting on VAN organized jointly by Britain's Royal Society and the International Council of Scientific Unions, where Varotsos and Seiya Uyeda, another leading VAN proponent who is based at both the University of Tokai and Texas A\&M University, put their case for VAN.

At the end of the meeting, according to one delegate, up to half of the participants ended up "sitting on the fence". The remainder were divided equally between supporters and sceptics. But the meeting is believed to have been boycotted by a number of Greek seismologists.

Sir James Lighthill, professor of mathematics at University College London, and the organizer of the meetings, says that its aim was to provide an impartial evaluation of VAN. The proceedings of the meeting are expected to be published by the end of the year.

Ehsan Masood 